\title{
High Percentage of ADAM-10 Positive Melanoma Cells Correlates with Paucity of Tumor-Infiltrating Lymphocytes but Does Not Predict Prognosis in Cutaneous Melanoma Patients
}

\author{
Piotr Donizy, ${ }^{1}$ Marcin Zietek, ${ }^{2}$ Marek Leskiewicz, ${ }^{3}$ \\ Agnieszka Halon, ${ }^{1}$ and Rafal Matkowski ${ }^{2,4}$ \\ ${ }^{1}$ Department of Pathomorphology and Oncological Cytology, Wroclaw Medical University, Borowska 213, 50-556 Wroclaw, Poland \\ ${ }^{2}$ Lower Silesian Oncology Centre, pl. Hirszfelda 12, 53-413 Wroclaw, Poland \\ ${ }^{3}$ Department of Statistics, Wroclaw University of Economics, Komandorska 118-120, 53-345 Wroclaw, Poland \\ ${ }^{4}$ Department of Oncology and Division of Surgical Oncology, Wroclaw Medical University, pl. Hirszfelda 12, 53-413 Wroclaw, Poland
}

Correspondence should be addressed to Piotr Donizy; piotrdonizy@wp.pl

Received 8 April 2015; Revised 6 July 2015; Accepted 8 July 2015

Academic Editor: Francesco A. Mauri

Copyright (C) 2015 Piotr Donizy et al. This is an open access article distributed under the Creative Commons Attribution License, which permits unrestricted use, distribution, and reproduction in any medium, provided the original work is properly cited.

\begin{abstract}
ADAM-10 (CDw156, CD156c, and kuzbanian) is a protein belonging to a superfamily of metalloproteases, enzymes capable of degrading the extracellular matrix. ADAMs have also been shown to be primarily involved in ectodomain cleavage. The aim of the study was to assess the expression and intracellular location of ADAM-10 in 104 primary skin melanomas and 16 metastatic lesions from regional lymph nodes. Also, prognostic significance of ADAM-10 expression in primary tumor cells and metastatic lesion cells was evaluated during 5-year observation. It was revealed that high expression of ADAM-10 positive cells was strictly related with lower intensity of tumor-infiltrating lymphocytes $(P=0.037)$, which suggests that ADAM-10 regulates immunoresponse in melanoma initiation and progression. No statistically significant correlations were found between ADAM-10 expression in primary tumor cells and nodal metastases and other histopathological parameters analyzed. Decreased immunoreactivity of ADAM-10 in cancer cells from regional lymph nodes was correlated with worse prognosis; however this correlation was statistically nonsignificant $(P=0.065)$. Review of the literature shows that our study is the first one ever to describe the significance of ADAM10 expression in correlation with detailed histopathological parameters of the primary tumor and data on long-term survival of cutaneous melanoma patients.
\end{abstract}

\section{Introduction}

ADAM-10 (CDw156, CD156c, and kuzbanian) is a protein belonging to a superfamily of metalloproteases, enzymes capable of degrading the extracellular matrix. ADAMs have also been shown to be primarily involved in ectodomain cleavage [1].

ADAM family proteins are membrane anchored glycoproteins which play a major role in a number of cytobiochemical processes such as proteolysis, intercellular adhesion, and activation of various signaling cascades $[2,3]$. The analysis of the molecular structure of ADAMs showed that they exhibit a few characteristic domains which, starting from their N-terminal ends, include a prodomain, a metalloprotease domain, a disintegrin-like domain, EGF-like domain, a cysteine-rich domain, and transmembrane and cytoplasmic fragments [4].

In a normal cell, catalytically active ADAM-10 protein functions as an enzyme shedding extracellular fragments of membrane-bound proteins (shedding), which leads to the release of active protein ectodomains. ADAM-10 also participates in the biochemical process referred to as regulated intramembrane proteolysis (RIP), during which, and with mediation by presenilin-dependent $\gamma$-secretase, intracellular domain (ICD) fragments are formed, which upon translocation to the nucleus modulate several signal transduction pathways and become either transcriptional activators or repressors $[5,6]$. A wide spectrum of protein substrates for ADAM-10 includes molecules that have been shown to be involved in carcinogenesis initiation and cancer progression. 
They include proteins such as CD44, E-cadherin, Notch, Delta, FasL, and TNF $\alpha$ and epidermal growth factor receptors [4].

The aim of the study was to assess the expression and intracellular location of ADAM-10 in 104 primary skin melanomas and 16 metastatic lesions from regional lymph nodes. Also, prognostic significance of ADAM-10 expression in primary tumor cells and metastatic lesion cells was evaluated during 5-year observation. Correlations were also analyzed between ADAM-10 immunoreactivity parameters and detailed clinicopathological parameters such as Breslow thickness and Clark level, presence of nodal and distant metastases, recurrence of primary tumor, mitotic rate, ulceration, lymphocytic inflammatory infiltration, regression, and microsatellitosis.

\section{Materials and Methods}

2.1. Patients. The study group consisted of 104 patients with CMM, who were diagnosed between 2005 and 2010 and treated in the Lower Silesian Oncology Center in Wroclaw, Poland. Additionally, tissue material obtained from 16 nodal metastatic foci was included in the study. The group was selected on the basis of tissue material (paraffin blocks and histopathology slides) and the availability of medical documentation. Comprehensive clinical data were obtained from archival medical records. The diagnostic and therapeutic procedures utilized were determined from medical records in the Oncology Outpatient Clinic of the Lower Silesian Oncology Center and data provided by the Lower Silesian Cancer Registry and Civil Register Office. The retrospective study was approved by the Ethical Committee of the Wroclaw Medical University, Poland.

Patients enrolled in the study were treated by thenavailable methods. Excisional biopsy of the primary lesion was performed. Once cutaneous melanoma was diagnosed in histopathological examination, the primary procedure was extended by excising the scar with a margin of 5,10 , or $20 \mathrm{~mm}$ of unaffected skin depending on Breslow thickness and primary tumor location, if any. If nodal metastases (cN0) were not clinically manifested and Breslow thickness was above $1 \mathrm{~mm}$ (>pTla), sentinel lymph node biopsy (SNLB) was performed. When metastases were observed in the regional lymph nodes (found either clinically or by SLNB), lymphadenectomy was performed.

Clinicopathological profile of patients included the following parameters: age and gender, primary tumor location, tumor stratification according to AJCC (pT), presence or absence of nodal $(\mathrm{pN})$ and distant $(\mathrm{pM})$ metastases, information on disease recurrence, and sentinel lymph node biopsy (SLNB) procedures (Table 1).

2.2. Tumor Samples and Histopathological Evaluation. Tumor specimens were fixed in $10 \%$ buffered formalin and embedded in paraffin. All haematoxylin and eosin stained sections were examined by two pathologists. The parameters of the primary tumor recorded in pathology reports were Breslow thickness, Clark level, growth phase, histologic type,
TABLE 1: Clinicopathological characteristics of the patients.

\begin{tabular}{lc}
\hline Clinicopathological characteristics & Number $(\%)$ \\
\hline All patients & $104(100.0)$ \\
\hline Age in years (21-79) & \\
Mean: 56.5 \pm 15.4; median: 58.5 & \\
Gender & $60(57.7)$ \\
Female & $44(42.3)$ \\
Male & \\
Primary tumor location & $15(14.4)$ \\
Head/neck & $18(17.3)$ \\
Upper extremity & $25(24.0)$ \\
Lower extremity & $42(40.4)$ \\
Trunk & $4(3.8)$ \\
Hand/foot & \\
Primary tumor (pT) & $34(32.7)$ \\
pT1 & $20(19.2)$ \\
pT2 & $27(26.0)$ \\
pT3 & $23(22.1)$ \\
pT4 & $60(57.7)$ \\
Sentinel lymph node biopsy status (SNLB) & $48(80.0)$ \\
No metastases (SNLB-) & $12(20.0)$ \\
Metastases present (SNLB+) & \\
Regional lymph nodes status (pN) & $86(82.7)$ \\
No metastases (pN-) & $18(17.3)$ \\
Metastases present (pN+) & \\
Recurrence & \\
No & \\
Yes & \\
Distant metastases & \\
No & \\
Yes & \\
\hline
\end{tabular}

mitotic rate (number of mitotic figures per $1 \mathrm{~mm}^{2}$ ), presence of ulceration, lymphangio invasion, microsatellitosis, intensity of lymphocytic inflammatory infiltrate (TILs: tumorinfiltrating lymphocytes), and microscopic evidence of regression (Table 2).

2.3. Immunohistochemistry. Formalin-fixed, paraffin embedded tissue was freshly cut $(4 \mu \mathrm{m})$. The sections were mounted on superfrost slides (Menzel Glaser, Germany), dewaxed with xylene, and gradually hydrated. The activity of endogenous peroxidase was blocked by 5 -minute exposure to $3 \%$ $\mathrm{H}_{2} \mathrm{O}_{2}$. The sections were boiled for 15 minutes at $250 \mathrm{~W}$ in Antigen Retrieval Solution (DakoCytomation, Denmark). Immunohistochemical reactions were then performed using polyclonal antibody detecting ADAM-10 (clone LS-B324, LifeSpan Biosciences, USA), N-cadherin (clone D-4, Santa Cruz Biotechnology, USA), and SPARC (clone PP16, Santa Cruz Biotechnology, USA). The tested sections were incubated with antibodies for 1 hour at room temperature. The subsequent incubations involved biotinylated antibodies (15 minutes at room temperature) and streptavidin-biotinylated peroxidase complex (15 minutes at room temperature) 
TABLE 2: Histopathological parameters of primary tumors.

\begin{tabular}{|c|c|}
\hline $\begin{array}{l}\text { Histopathological parameters of the primary } \\
\text { tumor }\end{array}$ & Number (\%) \\
\hline \multicolumn{2}{|l|}{ Breslow thickness } \\
\hline & $34(32.7)$ \\
\hline $1.01-2.00 \mathrm{~mm}$ & $20(19.2)$ \\
\hline \multirow[t]{2}{*}{$2.01-4.00 \mathrm{~mm}$} & $27(26.0)$ \\
\hline & $23(22.1)$ \\
\hline \multicolumn{2}{|l|}{ Clark level } \\
\hline I & $0(0.0)$ \\
\hline II & $18(17.3)$ \\
\hline III & $49(47.1)$ \\
\hline IV & $26(25.0)$ \\
\hline $\mathrm{V}$ & $11(10.6)$ \\
\hline \multicolumn{2}{|l|}{ Histologic type } \\
\hline Superficial spreading melanoma (SSM) & $68(65.4)$ \\
\hline Nodular malignant melanoma (NMM) & $32(30.8)$ \\
\hline Acral-lentiginous melanoma (ALM) & $4(3.8)$ \\
\hline \multicolumn{2}{|l|}{ Mitotic rate } \\
\hline 0 & $45(43.3)$ \\
\hline $1-2$ & $26(25.0)$ \\
\hline$\geq 3$ & $33(31.7)$ \\
\hline \multicolumn{2}{|l|}{ Ulceration } \\
\hline No & $55(52.9)$ \\
\hline Yes & $49(47.1)$ \\
\hline \multicolumn{2}{|l|}{ Lymphangio invasion } \\
\hline No & $74(71.2)$ \\
\hline Yes & $30(28.8)$ \\
\hline \multicolumn{2}{|l|}{ Growth phase } \\
\hline Radial & $3(2.9)$ \\
\hline Vertical & $101(97.1)$ \\
\hline \multicolumn{2}{|l|}{ Tumor-infiltrating lymphocytes (TILs) } \\
\hline No & $18(17.3)$ \\
\hline Nonbrisk & $34(32.7)$ \\
\hline Brisk & $52(50)$ \\
\hline \multicolumn{2}{|l|}{ Microsatellitosis } \\
\hline No & $98(94.2)$ \\
\hline Yes & $6(5.8)$ \\
\hline \multicolumn{2}{|l|}{ Tumor regression } \\
\hline No & $96(92.3)$ \\
\hline Yes & $8(7.7)$ \\
\hline
\end{tabular}

(LSAB+, HRP, DakoCytomation, Denmark). DAB (Vector Laboratories, UK) was used as a chromogen (10 minutes at room temperature). All sections were counterstained with Meyer's hematoxylin. In every case control reactions were performed, with the relevant antibody substituted by Primary Mouse Negative Control (DakoCytomation, Denmark).

2.4. Evaluation of Reaction Intensity. The intensity of the immunohistochemical reaction was estimated independently by two pathologists. Doubtful cases were reevaluated under a double-headed microscope and staining was discussed until consensus was achieved.

ADAM-10 expression was observed only in melanoma cells in both tissue material obtained from the primary tumor and nodal metastatic foci. No ALCAM immunoreactivity was identified in stromal compartment of tumor or lymphocytes from regional lymph nodes. Cancer cells of the primary tumors and metastatic cells displayed predominantly diffuse cytoplasmic expression.

The expression of ADAM-10 protein was calculated using a semiquantitative method. Two immunohistochemical reaction parameters were considered when evaluating the expression of the foregoing proteins: the percentage of cells with a positive cytoplasmic reaction (the percentage of reactive tissue) and the reaction intensity. The final immunohistochemical reaction results are expressed according to the semiquantitative IRS (ImmunoReactive Score) scale devised by Remmele and Stegner [7]. This scale assigns a score for the percentage of cells demonstrating reaction ( $0-4$ points) and for reaction intensity (0-3 points). The final result is the product of the scores for these two parameters ( $0-12$ points) and is referred to as an IRS factor or score.

2.5. Statistical Analysis. Statistical analysis was performed using the Statistica 10.0 and IBM SPSS 21 software packages. Overall survival (OS) was defined as the time between the primary surgical treatment and death, and OS was censored at last follow-up for patients who were still alive. A $\chi^{2}$ test, exact Fisher test in the case of $2 \times 2$ tables, and Spearman rank correlation were used to analyze associations between mitotic rate and the presence of ulceration and clinicopathological parameters. Differences between the means were tested with a nonparametric test (Mann-Whitney $U$ test and KruskalWallis test); the log-rank test was used to compare survival in two groups, the overall survival rate was estimated by the Kaplan-Meier method, and the influence of explanatory variables on death risk was analyzed by means of the Cox proportional hazard regression. $P$ values $<0.05$ were considered statistically significant.

\section{Results}

3.1. ADAM-10 Expression in Primary Tumors and Nodal Metastases. ADAM-10 expression defined as IRS $>0$ was found in 82 primary tumors (78.8\% of patients (Figure 1)). No ADAM-10 immunoexpression was revealed in 22 primary tumors $(21.2 \%)$. Average IRS was $4.08 \pm 3.04$. In nodal metastases ADAM-10 reactivity was shown in 9 out of 16 cases $(56.2 \%)$, and no expression (IRS $=0$ ) was found in 7 patients (43.8\%). Average IRS for nodal metastases was $3.50 \pm 4.31$.

3.2. Correlations between ADAM-10 Expression Parameters in Primary Tumor and Metastases with Histopathological Features of Primary Melanoma. It was revealed that high expression of ADAM-10 positive cells was strictly related with lower intensity of tumor-infiltrating lymphocytes $(P=0.037)$, 


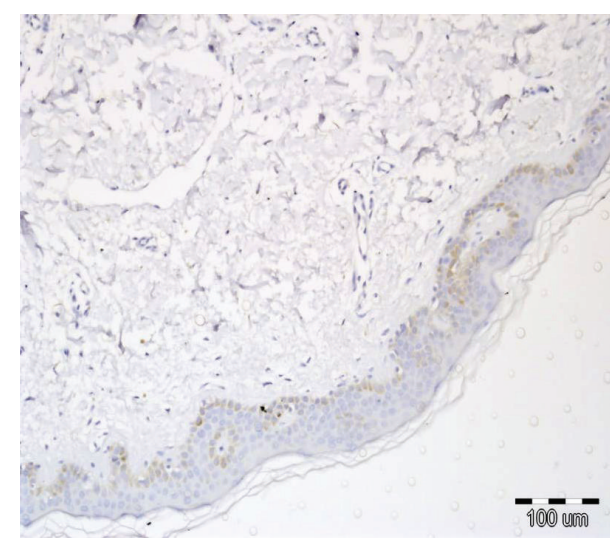

(a)

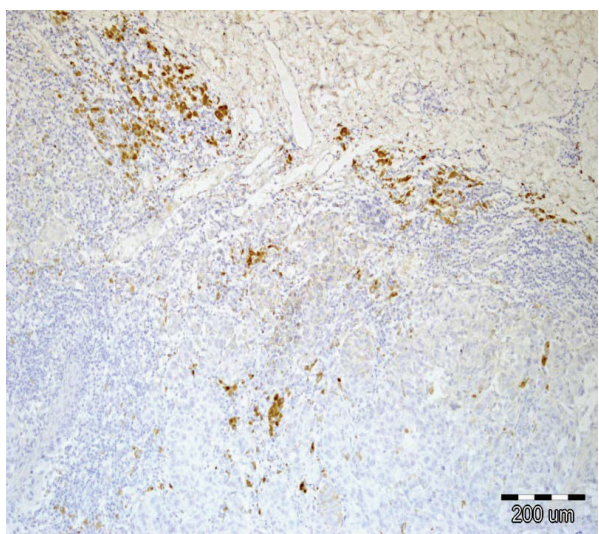

(c)

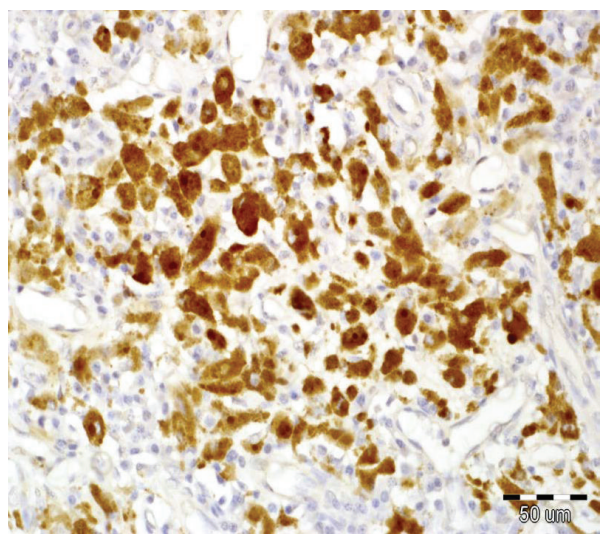

(e)

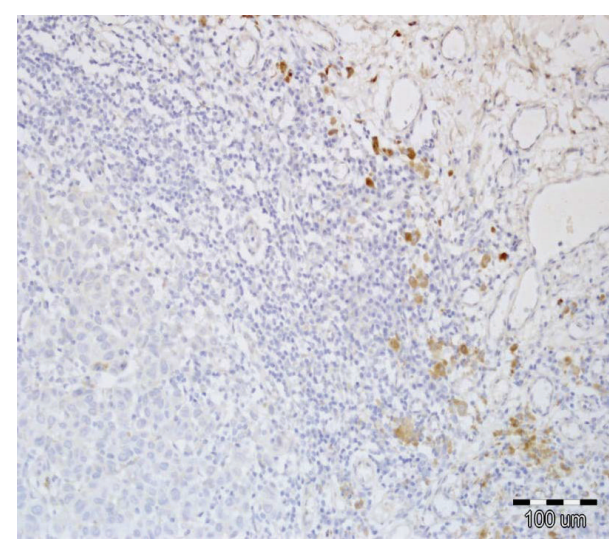

(b)

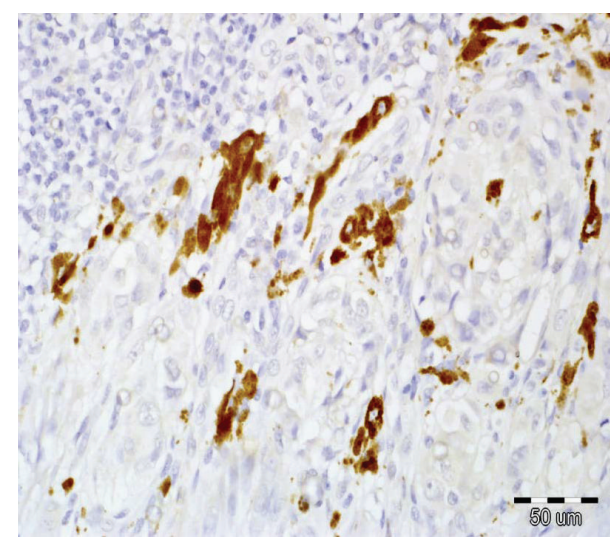

(d)

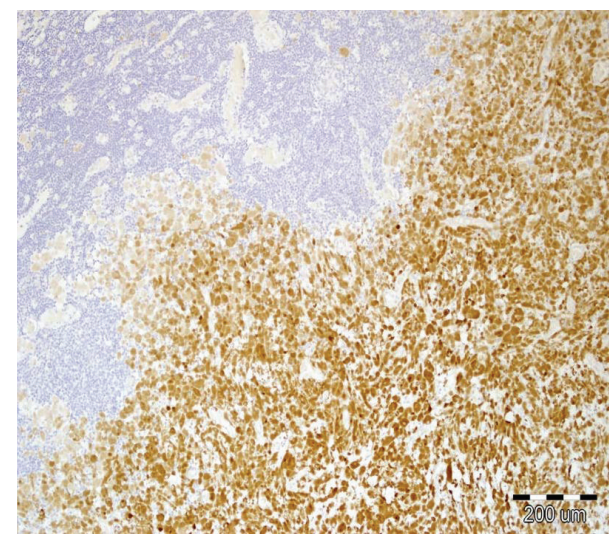

(f)

FIGURE 1: Immunohistochemically visualized expression of ADAM-10 in cutaneous melanoma. Lack of ADAM-10 immunoreactivity in normal skin ((a), 200x, hematoxylin). Low percentage of ADAM-10-positve cells in cutaneous melanoma with brisk tumor-infiltrating lymphocytes ((b), 10\% of ADAM-10 positive cells, 200x; (c), hematoxylin, 30\% of ADAM-10 positive cells, 200x, hematoxylin). Intermediate percentage of ADAM-10 positive cells in cutaneous melanoma with nonbrisk tumor-infiltrating lymphocytes ((d), 40\% of ADAM-10 positive cells, 400x). Enhanced ADAM-10 immunoreactivity in melanoma cells with scanty tumor-infiltrating lymphocytes ((e), IRS 12, 400x, hematoxylin). High cytoplasmic reactivity of ADAM-10 in regional lymph node metastases ((f), 200x, hematoxylin).

which suggests that ADAM-10 regulates immunoresponse in melanoma initiation and progression. No statistically significant correlations were found between ADAM-10 expression in primary tumor cells and nodal metastases and other histopathological parameters analyzed (Table 3 ).
3.3. Correlations between ADAM-10 Immunoreactivity and Clinicopathological Parameters. It was revealed that ADAM10 expression in primary tumor was reduced with increasing age at diagnosis $(P=0.029)$. Interestingly, lower ADAM-10 expression in cancer cells from nodal metastases 
TABLE 3: Correlations between clinicopathological and histopathological characteristics and ADAM-10 expression parameters in primary tumors and nodal metastases.

\begin{tabular}{|c|c|c|c|c|c|c|}
\hline \multirow[b]{2}{*}{ Clinicopathological parameters } & \multicolumn{3}{|c|}{ ADAM-10 expression, primary tumor } & \multicolumn{3}{|c|}{ ADAM-10 expression, nodal metastasis } \\
\hline & $\%$ & Int & IRS & $\%$ & Int & IRS \\
\hline $\mathrm{pT}^{\mathrm{a}}$ & 0.965 & 0.292 & 0.437 & 0.528 & 0.424 & 0.424 \\
\hline $\mathrm{pN}^{\mathrm{b}}$ & 0.195 & 0.757 & 0.820 & 0.262 & 0.273 & 0.477 \\
\hline Distant metastases $^{\mathrm{b}}$ & 0.292 & 0.088 & 0.204 & 0.482 & 0.673 & 0.934 \\
\hline Recurrence $^{\mathrm{b}}$ & 0.633 & 0.952 & 0.989 & 0.390 & 0.092 & 0.243 \\
\hline $\operatorname{Age}^{\mathrm{a}}$ & 0.785 & 0.145 & 0.029 & 0.043 & 0.037 & 0.037 \\
\hline Gender $^{\mathrm{b}}$ & 0.305 & 0.953 & 0.952 & 0.696 & 0.574 & 0.740 \\
\hline Primary tumor location $^{c}$ & 0.213 & 0.180 & 0.424 & 0.825 & 0.546 & 0.447 \\
\hline Clinicopathological parameters & $\%$ & Int & IRS & $\%$ & Int & IRS \\
\hline Breslow thickness ${ }^{\mathrm{a}}$ & 0.640 & 0.265 & 0.363 & 0.558 & 0.420 & 0.394 \\
\hline Clark level $^{\mathrm{a}}$ & 0.229 & 0.945 & 0.899 & 0.785 & 0.466 & 0.909 \\
\hline Growth phase ${ }^{b}$ & 0.614 & 0.264 & 0.220 & \multicolumn{3}{|c|}{$100 \%$ vertical growth phase } \\
\hline Histologic type $e^{c}$ & 0.462 & 0.299 & 0.160 & 0.390 & 0.500 & 0.342 \\
\hline Mitotic rate ${ }^{\mathrm{a}}$ & 0.319 & 0.367 & 0.552 & 0.845 & 0.140 & 0.248 \\
\hline Ulceration $^{\mathrm{b}}$ & 0.367 & 0.603 & 0.582 & 0.720 & 0.847 & 0.800 \\
\hline Lymphangio invasion $^{\mathrm{b}}$ & 0.290 & 0.531 & 0.485 & 0.631 & 0.205 & 0.212 \\
\hline Microsatellitosis $^{\mathrm{b}}$ & 0.398 & 0.275 & 0.551 & 0.631 & 0.300 & 0.307 \\
\hline $\begin{array}{l}\text { Tumor-infiltrating lymphocytes } \\
\text { (TILs) }^{c}\end{array}$ & 0.037 & 0.459 & 0.775 & 0.987 & 0.105 & 0.256 \\
\hline Tumor regression $^{\mathrm{b}}$ & 0.597 & 0.073 & 0.228 & \multicolumn{3}{|c|}{$100 \%$ without regression } \\
\hline
\end{tabular}

${ }^{\mathrm{a}} P$ value of Spearman rank correlation.

${ }^{\mathrm{b}} P$ value of Mann-Whitney $U$ test.

${ }^{\mathrm{c}} P$ value of Kruskal-Wallis test.

Statistically significant results $(P<0.05)$ are in bold font.

was also correlated with older age at diagnosis $(P=0.037)$ (Table 3).

Statistical analysis showed statistically nonsignificant trend of increased ADAM-10 immunoexpression in cancer cells from primary tumor and the presence of distant metastases $(P=0.088)$. Other clinical parameters such as $\mathrm{pT}$, $\mathrm{pN}$, recurrence, sex, and location of the primary tumor did not show any significant correlations with ADAM-10 expression (Table 3).

3.4. Analysis of ADAM-10 Expression Effect on 5-Year Survival in Melanoma Patients. Decreased immunoreactivity of ADAM-10 in cancer cells from regional lymph nodes was correlated with worse prognosis; however this correlation was statistically nonsignificant $(P=0.065)$. Other parameters of ADAM-10 expression in primary tumor and nodal metastasis cells did not have a statistically significant impact on the prognosis in cutaneous melanoma patients in the analyzed group of patients.

3.5. Analysis of the Correlations between ADAM-10 Expression Parameters and SPARC and N-Cadherin Immunoreactivity in Melanoma Cells. Statistical analysis revealed a significant correlation between enhanced immunoreactivity of ADAM10 and augmented expression of $\mathrm{N}$-cadherin and SPARC in primary tumor cells and melanoma cells in nodal metastases
(Table 4). N-Cadherin and SPARC are crucial proteins for the induction and progression of EMT (epithelial-mesenchymal transition) and these results may be associated with the potential role of ADAM-10 in the process of EMT.

\section{Discussion}

A review of the literature (PubMed, 1970-2015; keywords: ADAM-10 and melanoma) revealed only two articles that describe immunohistochemical analysis of ADAM-10 expression in cutaneous melanoma $[8,9]$.

Lee et al. [8] analyzed ADAM-10 immunoexpression based on 46 primary cutaneous melanomas and 127 melanoma metastases (106 carcinomas were distant organ metastases, and only 21 cases were nodal metastases). It must be stressed that the researchers did not assess ADAM-10 expression in the whole tumor but only in a section of tumor tissue using tissue microarrays (TMA) [8]. Lee et al. [8] observed no ADAM-10 expression in 35\% of primary tumors $(16 / 46)$, whereas in our study the percentage was $21.2 \%$ (22/104). Lee et al. [8] also showed no ADAM-10 expression in metastases in $26 \%$ of cases (33/127). In our study the percentage was $43.8 \%(7 / 16)$. It must be stressed that the data is not comparable or compatible since, in the metastatic carcinoma group studied by Lee et al. [8], distant organ metastases were the dominant ones (106/127), while nodal 
TABLE 4: Correlations between ADAM-10 expression parameters in primary tumors and nodal metastases and SPARC and N-cadherin immunoreactivity in melanoma cells in primary tumors and nodal metastases.

\begin{tabular}{|c|c|c|c|c|c|c|c|c|c|c|c|c|}
\hline \multirow[t]{2}{*}{$\begin{array}{l}\text { Expression of } \\
\text { ADAM-10 }\end{array}$} & \multicolumn{3}{|c|}{$\begin{array}{l}\text { Expression of SPARC } \\
\text { in primary tumor }\end{array}$} & \multicolumn{3}{|c|}{$\begin{array}{l}\text { Expression of SPARC } \\
\text { in nodal metastasis }\end{array}$} & \multicolumn{3}{|c|}{$\begin{array}{c}\text { Expression of } \\
\mathrm{N} \text {-cadherin in primary tumor }\end{array}$} & \multicolumn{3}{|c|}{$\begin{array}{l}\text { Expression of } \\
\mathrm{N} \text {-cadherin in nodal metastasis }\end{array}$} \\
\hline & $\%^{\mathrm{a}}$ & Int $^{\mathrm{b}}$ & IRS $^{c}$ & $\%$ & Int & IRS & $\%$ & Int & IRS & $\%$ & Int & IRS \\
\hline \multicolumn{13}{|l|}{ Primary tumor } \\
\hline$\%$ & 0.006 & 0.197 & 0.027 & 0.190 & 0.128 & 0.135 & 0.120 & 0.364 & 0.109 & 0.095 & 0.335 & 0.198 \\
\hline Int & $<0.001$ & $<0.001$ & $<0.001$ & 0.100 & 0.101 & 0.064 & $<0.001$ & $<0.001$ & $<0.001$ & 0.029 & 0.015 & 0.015 \\
\hline IRS & $<0.001$ & $<0.001$ & $<0.001$ & 0.010 & 0.041 & 0.007 & $<0.001$ & $<0.001$ & $<0.001$ & $<0.001$ & 0.003 & $<0.001$ \\
\hline \multicolumn{13}{|l|}{$\begin{array}{l}\text { Nodal } \\
\text { metastasis }\end{array}$} \\
\hline$\%$ & 0.193 & 0.149 & 0.128 & 0.001 & 0.066 & 0.004 & 0.393 & 0.060 & 0.212 & 0.004 & 0.024 & 0.004 \\
\hline Int & 0.107 & 0.279 & 0.124 & $<0.001$ & $<0.001$ & $<0.001$ & 0.288 & 0.052 & 0.110 & 0.022 & 0.001 & 0.002 \\
\hline IRS & 0.080 & 0.084 & 0.041 & $<0.001$ & $<0.001$ & $<0.001$ & 0.325 & 0.012 & 0.058 & 0.001 & $<0.001$ & $<0.001$ \\
\hline
\end{tabular}

metastases constituted only a small percentage (21/127; $16 \%)$. Our study was entirely focused on ADAM10 expression in cancer metastases from regional lymph nodes.

Similarly to our results, Lee et al. [8] did not show statistically significant correlations between Breslow thickness and ADAM-10 overexpression in cancer cells.

Lee et al. [8] showed that ADAM-10 immunoreactivity was significantly higher in metastases than in primary tumor $(P=0.04)$, which is not compatible with our results. Interestingly, our statistical analysis showed statistically nonsignificant trend of increased ADAM-10 immunoexpression in cancer cells from the primary tumor and the presence of distant metastasis $(P=0.088)$. Other clinical parameters such as $\mathrm{pT}$, $\mathrm{pN}$, sex, or location of the primary tumor were not shown to be significantly correlated with ADAM-10 expression.

It must be stressed that in our study it was only ADAM10 immunoreactivity in metastases that had prognostic value. Analysis of Kaplan-Meier curves showed that decreased expression of ADAM-10 in cancer cells from regional lymph node metastases was correlated with worse prognosis; however this correlation was statistically nonsignificant $(P=$ 0.065).

Anderegg et al. [9] analyzed ADAM-10 expression in melanoma cells only as regards its enzymatic activity related with CD44. 16 cases of melanoma and cell lines were analyzed in the paper, which confirmed that ADAM-10 was critically involved in enzymatic treatment of CD44 [9]. ADAM-10 influence on long-term survival or the correlation of ADAM10 expression with histopathological parameters of the primary tumor was not studied. Moreover, we also revealed a significant correlation between enhanced immunoreactivity of ADAM-10 and augmented expression of $\mathrm{N}$-cadherin and SPARC in primary tumor cells and melanoma cells in nodal metastases which may be associated with the potential role of ADAM-10 in the process of EMT $[10,11]$. It must be stressed that it is a hypothesis that needs to be supplemented and validated empirically with a much larger study group and animal models of metastasis; however, it is a voice in the discussion and confirms the hypotheses concerning the role of ADAM-10 in melanoma progression.

An innovative feature of our study was the finding that decreased intensity of tumor-infiltrating lymphocytes (TILs) was strictly correlated with the increased number of ADAM-10 positive cancer cells in the primary tumor $(P=$ 0.037). The possible meaning of this correlation is unclear and needs further studies, yet it suggests that ADAM-10 is involved in the regulation of immunological response in the initiation and progression of melanoma. Neither of the research teams described in the quoted papers analyzed or correlated ADAM-10 expression parameters with the details of the histopathology report $[8,9]$. Furthermore, it was shown that ADAM-10 expression in the primary tumor $(P=$ $0.029)$ was reduced with increasing age at diagnosis and that decreased expression of ADAM-10 in cancer cells from nodal metastases was also correlated with more advanced age at diagnosis $(P=0.037)$. Lee et al. [8] and Anderegg et al. [9] did not study the potential impact of age on ADAM-10 expression in cancer cells.

To sum up, it must be pointed out that, in the two papers referred to above by Lee et al. [8] and Anderegg et al. [9], only the expression patterns of ADAM-10 were studied and the impact on long-term survival was not analyzed. Review of the literature shows that our study is the first one ever to describe the significance of ADAM-10 expression in correlation with detailed histopathological parameters of the primary tumor and data on long-term survival of cutaneous melanoma patients.

\section{Conflict of Interests}

The authors declared no potential conflict of interests with respect to the research, authorship, and/or publication of this paper. 


\section{Authors' Contribution}

Piotr Donizy and Marcin Zietek contributed equally to the paper.

\section{Acknowledgment}

This study was supported by Wroclaw Medical University research Grant ST-843 and Pbmn157.

\section{References}

[1] S. Mochizuki and Y. Okada, "ADAMs in cancer cell proliferation and progression," Cancer Science, vol. 98, no. 5, pp. 621-628, 2007.

[2] D. R. Edwards, M. M. Handsley, and C. J. Pennington, "The ADAM metalloproteinases," Molecular Aspects of Medicine, vol. 29, no. 5, pp. 258-289, 2008.

[3] C. P. Blobel, "ADAMs: key components in egfr signalling and development," Nature Reviews Molecular Cell Biology, vol. 6, no. 1, pp. 32-43, 2005.

[4] H. C. Crawford, P. J. Dempsey, G. Brown, L. Adam, and M. L. Moss, "ADAM10 as a therapeutic target for cancer and inflammation," Current Pharmaceutical Design, vol. 15, no. 20, pp. 2288-2299, 2009.

[5] D. F. Seals and S. A. Courtneidge, "The ADAMs family of metalloproteases: multidomain proteins with multiple functions," Genes and Development, vol. 17, no. 1, pp. 7-30, 2003.

[6] M. S. Wolfe and R. Kopan, "Intramembrane proteolysis: theme and variations," Science, vol. 305, no. 5687, pp. 1119-1123, 2004.

[7] W. Remmele and H. E. Stegner, "Recommendation for uniform definition of an immunoreactive score (IRS) for immunohistochemical estrogen receptor detection (ER-ICA) in breast cancer tissue," Pathologe, vol. 8, no. 3, pp. 138-140, 1987.

[8] S. B. Lee, A. Schramme, K. Doberstein et al., "ADAM10 is upregulated in melanoma metastasis compared with primary melanoma," Journal of Investigative Dermatology, vol. 130, no. 3, pp. 763-773, 2010.

[9] U. Anderegg, T. Eichenberg, T. Parthaune et al., "ADAM10 is the constitutive functional sheddase of CD44 in human melanoma cells," Journal of Investigative Dermatology, vol. 129, no. 6, pp. 1471-1482, 2009.

[10] G. Musumeci, R. Coleman, R. Imbesi et al., "ADAM-10 could mediate cleavage of $\mathrm{N}$-cadherin promoting apoptosis in human atherosclerotic lesions leading to vulnerable plaque: a morphological and immunohistochemical study," Acta Histochemica, vol. 116, pp. 1148-1158, 2014.

[11] S. Paudel, Y.-H. Kim, M.-I. Huh et al., "ADAM10 mediates Ncadherin ectodomain shedding during retinal ganglion cell differentiation in primary cultured retinal cells from the developing chick retina," Journal of Cellular Biochemistry, vol. 114, no. 4, pp. 942-954, 2013. 


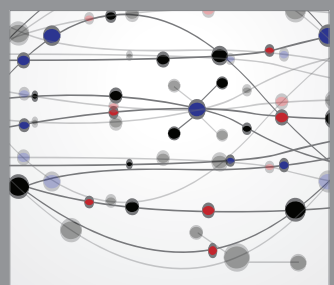

The Scientific World Journal
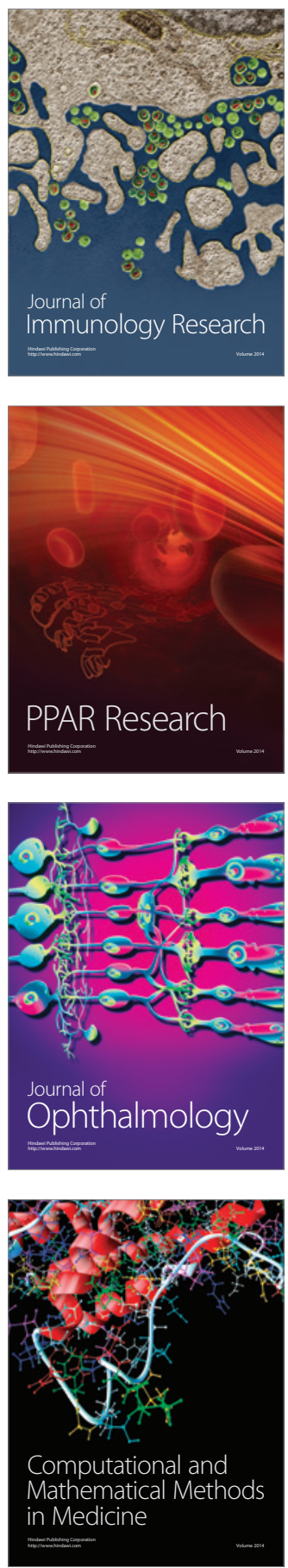

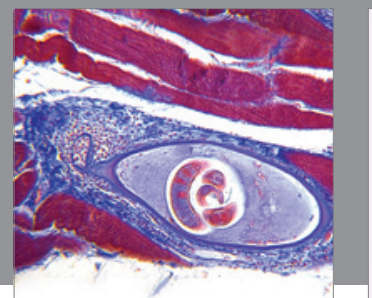

Gastroenterology

Research and Practice
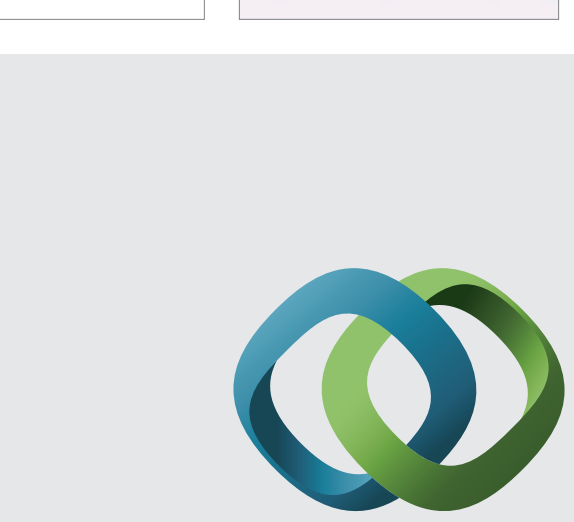

\section{Hindawi}

Submit your manuscripts at

http://www.hindawi.com
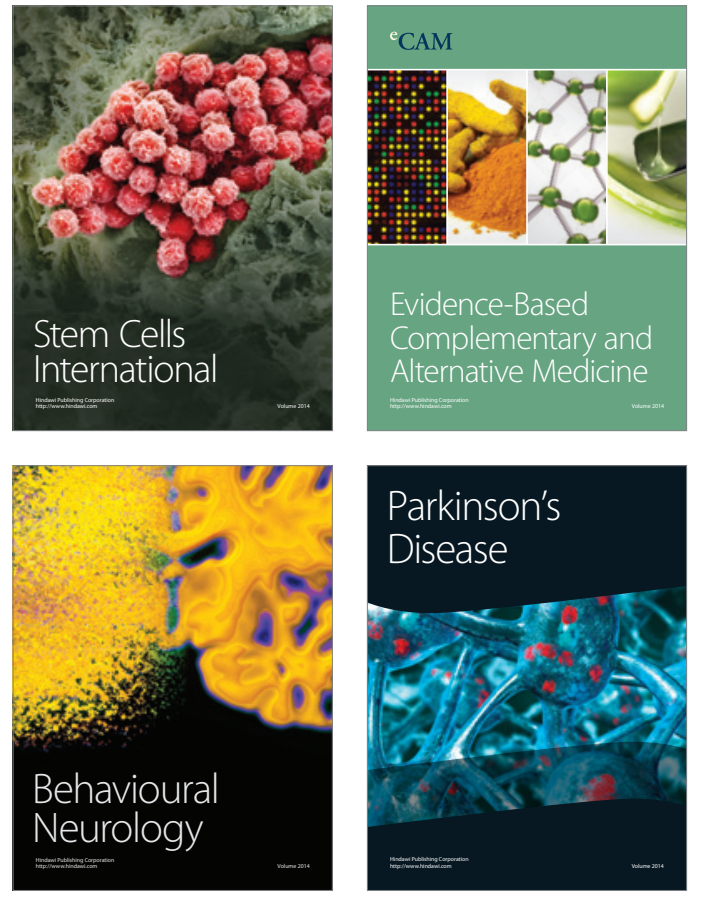
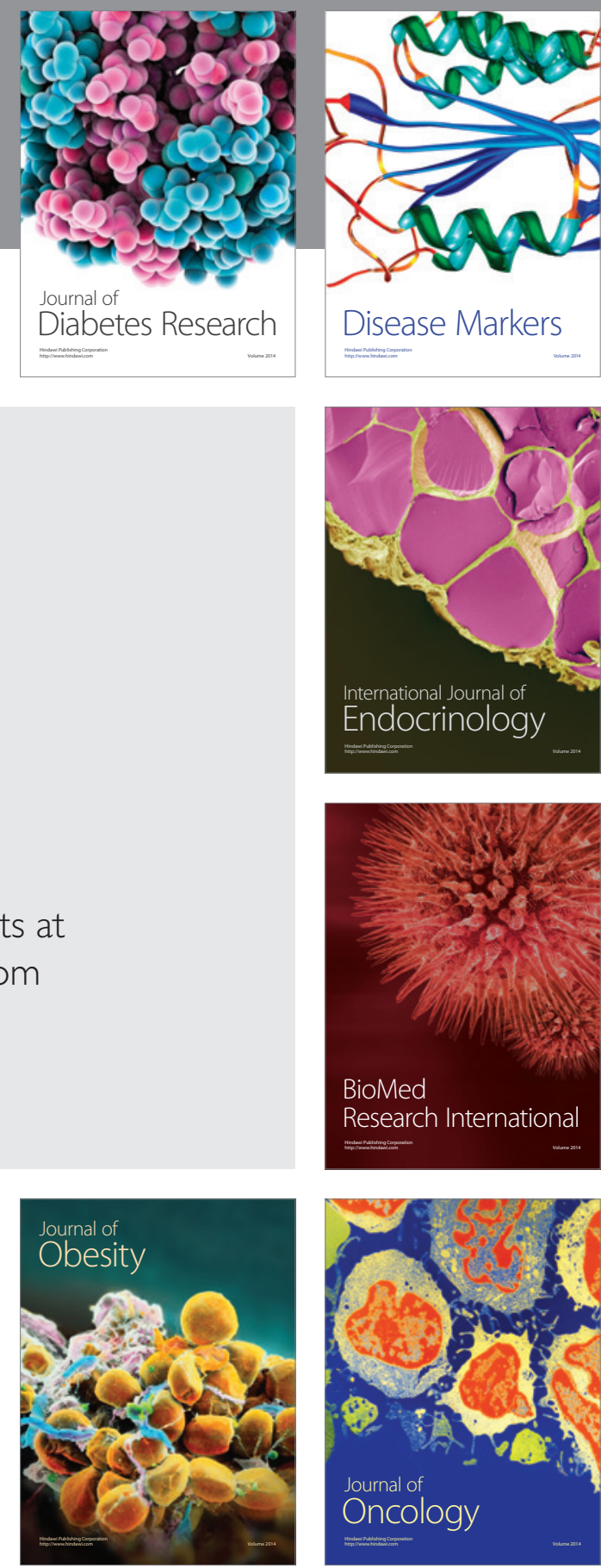

Disease Markers
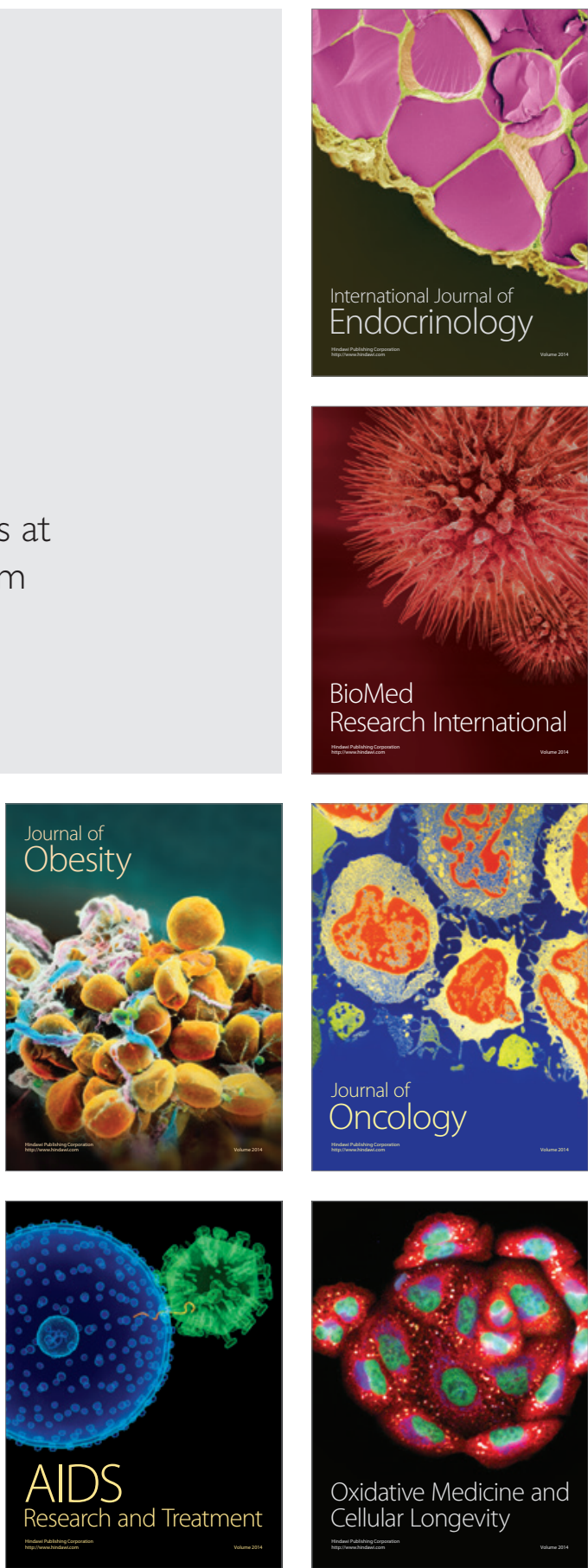